\title{
Risk of acute rejection in kidney transplant recipients after COVID-19
}

\author{
Enzo Vásquez-Jiménez ${ }^{1,2}\left([) \cdot\right.$ Bernardo Moguel-González $^{1,3} \cdot$ Virgilia Soto-Abraham $^{4,5}\left({ }^{(0)} \cdot\right.$ César Flores-Gama $^{1}(\mathbb{0}$
}

Received: 27 September 2021 / Accepted: 22 October 2021 / Published online: 17 November 2021

(C) Italian Society of Nephrology 2021

Keywords COVID-19 $\cdot$ Kidney transplant $\cdot$ Acute rejection $\cdot$ Subclinical rejection $\cdot$ Donor specific antibodies

There is global concern about outcomes after COVID-19 in kidney transplant recipients. To date, large cohort studies have shown higher rates of AKI and mortality in kidney transplant recipients who developed COVID-19 than in the general population, however it is still debated whether the immunological response associated with SARS-CoV-2 infection and/or the immunosuppressive modifications increase the risk of rejection $[1,2]$.

Since the beginning of the COVID-19 pandemic, the decrease in, and withdrawal of immunosuppressors, particularly in severe cases, has been a common practice. However, these strategies are not risk-free [3,4].

We evaluated the presence of de novo donor specific antibodies (dnDSAs) and kidney biopsies in a group of kidney transplant recipients after recovering from COVID19. Twenty kidney transplant recipients followed-up at the National Institute of Cardiology in Mexico City, with a follow-up of at least 4 weeks after COVID-19 diagnosis, and with eGFR $>20 \mathrm{ml} / \mathrm{min} / 1.73 \mathrm{~m}^{2}$ before COVID-19 diagnosis were included. Four weeks after COVID-19 diagnosis, antiHLA antibodies and kidney graft biopsy were performed (Fig. S1).

César Flores-Gama

cesarfloresgama@gmail.com

1 Department of Nephrology, National Institute of Cardiology Ignacio Chavez, Juan Badiano No. 1, Col. Sección XVI, Tlalpan, 14080 Mexico City, Mexico

2 Department of Nephrology, ISSEMYM Medical Center, Toluca, Mexico

3 Department of Interventional Nephrology, National Institute of Cardiology Ignacio Chavez, Mexico City, Mexico

4 Department of Pathology, National Institute of Cardiology Ignacio Chavez, Mexico City, Mexico

5 Department of Pathology, General Hospital of Mexico, Eduardo Liceaga, Mexico City, Mexico
Detection and characterization of anti-HLA antibodies were performed using Single Antigen Flow Beads assays (LSA class I and class II, Immucor, Norcross, GA). Luminex mean fluorescence intensity (MFI) was measured on a LABscan IS 200, specificities with an MFI $\geq 1000$ were considered positive. De novo DSAs (dnDSAs) were considered positive when they had not been identified pre-transplantation. Kidney biopsy was planned 4 weeks after COVID-19 diagnosis, however, some biopsies had to be deferred. All biopsies were analyzed by a single expert kidney pathologist. Histological lesions were classified according to The Banff 2019 Kidney Meeting Report [5].

The baseline characteristics of kidney recipients are shown in Table 1. The details concerning clinical presentation are shown in Table S1. In our center, immunosuppressive treatment was decreased or withdrawn in $60 \%$ of patients, and excluding 3 cases, all patients had returned to their usual immunosuppressive regimen at the time of biopsy. We did not find a different pattern of immunosuppressive regimen modification in patients with and without rejection ( 67 vs $57 \%, P=0.33$ ).

Thirty percent of patients had no major abnormalities in their kidney biopsy, 20\% had chronic active antibodymediated rejection (ABMR), 15\% active ABMR, 20\% mixed ABMR/ T cell mediated rejection (TCMR), $10 \%$ borderline for acute TCMR, and 5\% chronic active TCMR (Table S2). All patients who developed dnDSAs $(n=11)$ were diagnosed with rejection, 27.2\% with ABMR, 36.4\% mixed ABMR/ TCMR and $36.4 \%$ with chronic ABMR.

Among cases diagnosed with rejection, $57 \%$ were considered subclinical. Subclinical rejection was diagnosed in all cases borderline for active TCMR and active ABMR, in $50 \%$ of active chronic ABMR, and in $25 \%$ of mixed ABMR/ TCMR, while all TCMR and $16.7 \%$ of biopsies with no major abnormalities had persistent kidney injury at biopsy. A detailed description is available in Tables 2 and S3. 
Table 1 Baseline characteristics of kidney recipients

\begin{tabular}{|c|c|c|c|c|}
\hline & $\begin{array}{l}\text { Total } \\
(n=20)\end{array}$ & $\begin{array}{l}\text { Without histological } \\
\text { sign of rejection }(n=6)\end{array}$ & $\begin{array}{l}\text { With histological } \\
\text { sign of Rejection } \\
(n=14)\end{array}$ & $P$ value \\
\hline Age, years & $32.5(30.5-37.5)$ & $32(31-38)$ & $34(30-37)$ & 0.97 \\
\hline Females & $11(55)$ & $1(16.7)$ & $10(71.4)$ & 0.04 \\
\hline BMI, $\mathrm{kg} / \mathrm{m}^{2}$ & $27 \pm 6.4$ & $23.9 \pm 4.9$ & $28.5 \pm 6.6$ & 0.14 \\
\hline CKD Etiology & & & & 0.68 \\
\hline Unknown & $17(85)$ & $5(83.3)$ & $12(85.7)$ & \\
\hline Other & $3(15)$ & $1(16.7)$ & $2(14.3)$ & \\
\hline Diabetes & $2(10)$ & $2(33.3)$ & $0(0)$ & 0.08 \\
\hline Hypertension & $5(25)$ & $1(16.7)$ & $4(28.6)$ & 0.52 \\
\hline Transplant vintage, months & $60(12.5-110.5)$ & $33(7-75)$ & $66.5(26-139)$ & 0.32 \\
\hline Deceased donor & $8(40)$ & $2(33.3)$ & $6(42.9)$ & 0.55 \\
\hline Retransplant & $2(10)$ & $0(0)$ & $2(14.3)$ & 0.48 \\
\hline Pretransplant PRA I, \% & $0(0-3)$ & $0(0-4)$ & $0(0-2)$ & 0.85 \\
\hline Pretransplant PRA II, \% & $0(0-4)$ & $1.5(0-6)$ & $0(0-2)$ & 0.59 \\
\hline Preexisting DSA & $7(35)$ & $3(50)$ & $4(28.6)$ & 0.34 \\
\hline Induction & & & & 0.3 \\
\hline MPD alone & $4(20)$ & $1(16)$ & $3(21.4)$ & 0.66 \\
\hline MPD + Inh IL-2r & $11(55)$ & $2(33.3)$ & $9(64.3)$ & 0.22 \\
\hline MPD + Thymoglobulin & $5(25)$ & $3(50)$ & $2(14.3)$ & 0.13 \\
\hline Maintenance & & & & 1 \\
\hline $\mathrm{TAC}+\mathrm{MMF}+\mathrm{PD}$ & $15(75)$ & $5(83.3)$ & $10(71.4)$ & \\
\hline $\mathrm{CyA}+\mathrm{MMF}+\mathrm{PD}$ & $2(10)$ & $1(16.7)$ & $1(7.1)$ & \\
\hline $\mathrm{TAC}+\mathrm{AZT}+\mathrm{PD}$ & $2(10)$ & $0(0)$ & $2(14.3)$ & \\
\hline $\mathrm{AZT}+\mathrm{PD}$ & $1(5)$ & $0(0)$ & $1(7.1)$ & \\
\hline Previous rejection & $6(30)$ & $2(33.3)$ & $4(28.6)$ & 0.61 \\
\hline Previous rituximab & $6(31.6)$ & $1(20)$ & $5(35.7)$ & 0.48 \\
\hline Baseline $\mathrm{Cr}, \mathrm{mg} / \mathrm{dl}$ & $1.6(1.2-2)$ & $1.5(1.3-2.1)$ & $1.7(1.1-1.9)$ & 0.84 \\
\hline Non-adherence & $5(25)$ & $1(16.7)$ & $4(28.6)$ & 0.52 \\
\hline
\end{tabular}

Values stated in $n(\%)$, median $(25-75 \%)$ or mean \pm sd

$A Z T$ azathioprine, $B M I$ body mass index, DSA donor-specific antibodies, $C K D$ chronic kidney disease, $M M F$ mycophenolate mofetil, $M P D$ methylprednisolone, $P D$ prednisone, $P R A$ panel reactive antibody, TAC tacrolimus
We found that $70 \%$ of patients who recovered from COVID-19 had signs of acute rejection in the kidney graft biopsy. This high rate of biopsy-proven signs of rejection, almost half of which are classified as subclinical rejections, is a matter of concern. In a cohort of 47 kidney transplant recipients with immunosuppression minimization for COVID-19, Pampols et al. reported that none developed dnDSAs; however, allograft biopsies were not performed [6].

Six of our patients had a history of acute rejection, in 3 of them the allograft biopsy revealed chronic active ABMR, which may be the evolution of the previous rejection. However, even excluding these patients, biopsy revealed active rejection in 9 patients without a history of rejection. It is possible that dnDSAs were present before the COVID-19 diagnosis, however $25 \%$ of acute rejection type 2 were diagnosed within 12 months after transplantation, increasing the chance that dnDSAs were developed close to COVID-19. As for adherence to immunosuppressive treatment during the SARS-CoV-2 pandemic, Aziz et al. reported on kidney recipients without a diagnosis of COVID-19 who developed acute rejection during the COVID-19 pandemic due to non-adherence and loss to follow-up [7]. This possibility cannot be ruled out in our series.

Our analysis is preliminary, and the lack of serial biopsies and dnDSAs tests does not allow drawing cause-effect conclusions; however, within these limits, our findings suggest that COVID-19-related immunologic challenge, together with the reduction of immunosuppresion may trigger kidney transplant rejection; this should be a warning to transplant centers to monitor allograft dysfunction. Nonetheless, stable serum creatinine after COVID-19 infection does not exclude ongoing damage to the graft, therefore, a kidney 
Table 2 Characteristics at kidney graft biopsy and pathological diagnosis

\begin{tabular}{lc}
\hline Patients & $n=20$ \\
\hline Months after COVID-19 & $2(1.4-3.5)$ \\
sCr at biopsy, mg/dL & $1.5(1.4-2.4)$ \\
$\Delta$ sCr, basal-biopsy, mg/dL & $0.7(0.2-1.3)$ \\
$\Delta$ sCr, COVID diagnosis-biopsy, mg/dL & $0.28(-1.1$ to +0.02$)$ \\
Persistent kidney dysfunction & $7(35)$ \\
Tacrolimus, ng/ml & $6.4(5.2-7.6)$ \\
PRA I, \% & $4(0-17)$ \\
PRA II, \% & $10(2-19)$ \\
De novo DSA & $11(55)$ \\
Class I & $2(10)$ \\
Class II & $6(30)$ \\
Class I \& II & $3(15)$ \\
Kidney biopsy & \\
Glomeruli & $21(10-27)$ \\
Diagnosis & \\
No major abnormalities & $6(30)$ \\
Borderline for acute TCMR & $2(10)$ \\
Chronic active TCMR & $1(5)$ \\
Active ABMR & $3(15)$ \\
Mixed ABMR/TCMR & $4(20)$ \\
Chronic active ABMR & $4(20)$ \\
\hline
\end{tabular}

Values stated in $n(\%)$, median (25-75\%) or mean $\pm \mathrm{sd}$

$\Delta$ delta, ABMR antibody mediated rejection, COVID-19 coronavirus disease-19, $s C r$ serum creatinine, DSA donor-specific antibodies, $M M F$ mycophenolate mofetil, PRA panel reactive antibody, TCMR T-cell mediated rejection

biopsy should be considered. Further studies are needed to confirm these concerning findings.

Supplementary Information The online version contains supplementary material available at https://doi.org/10.1007/s40620-021-01192-x.

Acknowledgements We thank the Nephrology medical staff from the National Institute of Cardiology.

Author contributions EVJ: investigation, writing-review \& editing; BMG: investigation, review \& editing; VSA: kidney-graft biopsy analysis, review \& editing; CFG: investigation, writing, methodology, review \& editing.
Funding This research received no specific grant from any funding agency from any sector.

\section{Declarations}

Conflict of interest All the authors declare no competing interests.

Ethical statement The study was approved by the National Institute of Cardiology Ethics Board (approval number (21-1209).

Informed consent Written informed consent was obtained from all study patients.

\section{References}

1. Batlle D, Soler MJ, Sparks MA, Hiremath S, South AM, Welling PA et al (2020) Acute kidney injury in COVID-19: emerging evidence of a distinct pathophysiology. J Am Soc Nephrol 31:1380-1383

2. Gabarre P, Dumas G, Dupont T, Darmon M, Azoulay E, Zafrani L (2020) Acute kidney injury in critically ill patients with COVID19. Intensive Care Med 46:1339-1348

3. Michaels MG, La Hoz RM, Danziger-Isakov L, Blumberg EA, Kumar D, Green M et al (2020) Coronavirus disease 2019: implications of emerging infections for transplantation. Am J Transplant 20:1768-1772

4. Mahalingasivam V, Craik A, Tomlinson LA, Ge L, Hou L, Wang Q et al (2021) A systematic review of COVID-19 and kidney transplantation. Kidney Int Rep 6:24-45

5. Loupy A, Haas M, Roufosse C, Naesens M, Adam B, Afrouzian $M$ et al (2020) The banff 2019 kidney meeting report (I): updates on and clarification of criteria for T cell- and antibody-mediated rejection. Am J Transplant 20:2318-2331

6. Pampols PA, Trujillo H, Melilli E, Urban B, Sandino J, Favá A et al (2021) Immunosuppression minimization in kidney transplant recipients hospitalized for COVID-19. Clin Kidney J 14:1229-1235

7. Aziz F, Muth B, Parajuli S, Garg N, Mohamed M, Mandelbrot $M$ et al (2020) Unusually high rates of acute rejection during the COVID-19 pandemic: cause for concern? Kidney Int 98:513-514

Publisher's Note Springer Nature remains neutral with regard to jurisdictional claims in published maps and institutional affiliations. 